Linguistique, littérature, didactique

191-192 | 2021

Théâtre contemporain : Écritures et Représentations

\title{
Emmanuelle PRAK-DERRINGTON, Magies de la répétition
}

Lyon, ENS Éditions, 2021

\section{Michel Favriaud}

\section{(2) OpenEdition}

\section{Journals}

Édition électronique

URL : https://journals.openedition.org/pratiques/10959

DOI : 10.4000/pratiques.10959

ISSN : 2425-2042

Éditeur

Centre de recherche sur les médiations (CREM)

Référence électronique

Michel Favriaud, «Emmanuelle prak-Derrington, Magies de la répétition», Pratiques [En ligne], 191-192 |

2021, mis en ligne le 15 décembre 2021, consulté le 04 janvier 2022. URL : http://

journals.openedition.org/pratiques/10959; DOI : https://doi.org/10.4000/pratiques.10959

Ce document a été généré automatiquement le 4 janvier 2022.

(c) Tous droits réservés 


\title{
Emmanuelle PRAK-DERRINGTON, Magies de la répétition
}

Lyon, ENS Éditions, 2021

\author{
Michel Favriaud
}

1 Ce gros livre de linguistique, publié aux Éditions de l'ENS en janvier 2021 par E. PrakDerrington, enseignante-chercheure à l'École normale supérieure (ENS) de Lyon, dans la discipline de linguistique et de stylistique allemandes, a de quoi surprendre, enchanter, ouvrir des débats.

2 D'abord par son titre, qui ne s'adresse pas seulement aux spécialistes de la spécialité, mais qui associe un phénomène linguistique, ou plutôt discursif, « la répétition », à une notion plutôt populaire, "magies » (surtout fléchie au pluriel), ou ethnographique : de fait, ce livre est lisible par tous ceux qui s'intéressent à la langue, ou plutôt aux langues, française, allemande et anglaise, et fait pont avec les sciences sociales, dans un beau langage explicite.

3 Ensuite par son préfacier célèbre, $C$. Hagège, dont le nom vient en dessous du nom de l'auteur sur la première de couverture, lequel ne ménage pas les compliments à son auteure, au risque de ne pas ouvrir le débat.

4 Enfin par ses dédicace et remerciements familiaux et amicaux, habituels dans une thèse, mais qui ici suggèrent peut-être que le sujet traité est aussi une affaire personnelle, éthique, ce qu'on oublie souvent en linguistique et dans les sciences sociales : l'imaginaire singulier et l'éthos personnel peuvent faire bon ménage avec la rigueur scientifique; le corpus lui-même ne sera ni neutre, ni quantitatif, mais constitué par des prélèvements personnels, un peu dans des œuvres littéraires des trois langues, beaucoup dans des discours politiques célèbres, dans des films du grand répertoire et dans des conversations orales; l'auteure revendique souvent la position de l'honnête homme et de la femme honnête, consultant d'abord la pile des grands dictionnaires et voulant ostensiblement ouvrir sa réflexion sur des discours " épidictiques », ou religieux. 
5 Mais sous ce titre légèrement racoleur, ou peut-être roué, sous cet appareil en apparence hétérogène se cache une ambition immense: faire apparaître dans les "discours » les plus répandus un autre fonctionnement de la langue, peut-être nonconforme à nos idées reçues, peut-être un chamboule-tout qui se situerait dans le prolongement théorique du néo-saussurisme, et de ce qu'on pourrait appeler le néobenvenistisme, celui du Baudelaire, livre inachevé d'É. Benveniste, publié il y a peu chez Lambert-Lucas. C'est le rapport existentiel au langage, «la vie dans le langage » qui seraient en jeu, au-delà des théories de la signifiance (É. Benveniste) et de l'énonciation (O. Ducrot, J. Authier-Revuz, A. Rabatel) qui sont pourtant les matrices théoriques de base de cette étude.

6 Le prologue ouvre le champ très largement, chaque lecteur jugera si c'est trop. La répétition ne serait pas seulement un phénomène linguistico-discursif mais une sorte d'ADN universel, structurant tout le vivant et le cosmique. Les deux épigraphes initiales montrent bien l'ampleur de cet empan :

« Le soleil est nouveau chaque jour » d'Héraclite et : «Si Dieu n’avait pas souhaité la répétition, le monde n'aurait jamais été créé » de $\mathrm{S}$. Kierkegaard.

Rien moins que la création du monde et le soleil, avec Dieu en surplomb possible. En suggérant néanmoins - ce qui sera un des principes bien mis au jour, au demeurant reconnu depuis longtemps par les poéticiens - que la répétition est à la fois retour du même, variation et amplification, et, pour nous ici, nouveauté et différentialité sémantique. On pressent alors que l'auteur ne prend guère le parti du premier $F$. de Saussure, celui du Cours de linguistique générale retranscrit par C. Bally et A. Séchehaye, où le Genevois voulait, pour fonder la linguistique comme science, et presque comme science mathématisable, couper le cordon de la linguistique, d'une part, et de l'ontologie et de la métaphysique, d'autre part. Notre auteure veut au contraire retisser les liens, problématiques, non seulement avec la littérature (et peut-être la poésie), mais avec les sciences, la philosophie, voire les religions, donc entre le corps et l'esprit.

Cela aboutit parfois, et dès le début, à des affirmations qui ne sont peut-être pas évidentes pour tous, comme :

À l'instar du «temps» ou de la «personne » qui sont pour tout un chacun des données universelles de l'expérience humaine avant d'être des catégories linguistiques, le mot "répétition" renvoie à des phénomènes extrêmement hétérogènes, qui vont bien au-delà du langage. (p. 15)

La comparaison entre temps/personne et répétition ne s'impose guère, même si l'auteure va inscrire la répétition dans une théorie philosophique du temps. La "personne » existe-t-elle avant d'être une catégorie du langage est une question pendante (on pourrait plaider pour une co-construction), sachant que d'une langue à l'autre elle est marquée différemment, peu en chinois semble-t-il, sachant aussi que l'allocutaire aura une forme de politesse différente en français standard, en français de chalandise, en allemand, en anglais, etc. La seconde question posée tant par les dédicaces que par cette entrée est de savoir si sous ce mot de "répétition » ne se cachent pas des realia radicalement différents ("phénomènes extrêmement hétérogènes ", admet l'auteure). Parle-t-on de la même chose quand on parle de « répétition » d'une structure d'ADN (p. 17) et de « répétition » linguistique ? Parle-t-on de la même chose en langue et discours quand on parle, d'un côté, de la duplication des graphèmes/phonèmes de l'orthographe et de la phonologie communes et, de l'autre, de la répétition intentionnelle de mots, de structures syntaxiques? Si on regarde du côté 
de la ponctuation, la ponctuation noire notamment: parlera-t-on de répétition des points finals de phrase, ou des virgules, marqueurs qui sont pourtant traités comme des signes par la plupart des linguistes ? Peut-être faudrait-il faire la différence entre « répétition» et «duplication», ce dernier terme plus en usage dans les sciences du vivant et de la matière.

On pourra aussi taquiner l'auteure sur l'usage des trois langues et leur statut dans cet ouvrage, l'allemand seul étant traduit, alors que l'anglais est dit lingua franca. On peut ne pas être d'accord avec ce principe, surtout quand l'auteure affiche une volonté d'ouvrir la réflexion au plus grand nombre. Si l'on poussait ce principe au bout, il faudrait tout écrire en anglais, ce qui serait bien dommage car le français de l'auteure est de valeur, et ses références théoriques sont, en dehors de la théorie de la Gestalt et des sciences cognitives, largement francophones. Il y aurait peut-être chez elle - qui sait ? - une façon de dire, de faire, de démontrer a la francesca... Cum grano salis.

11 Ces questions toutefois ne doivent pas nous faire perdre de vue l'essentiel: c'est qu'E. Prak-Derrington, cherche, avec l'aide, ou la caution, des sciences et de la philosophie, à mettre au jour un principe linguistico-discursif de grande ampleur, qui outrepasse le petit tiroir des onomatopées, lallations enfantines et autres tours de la "réduplication», ou celui plus englobant de la répétition avec, notamment, ses multiples déclinaisons stylistiques en anaphore, chiasme, anadiplose, etc. La répétition au sens large serait, au même titre que l'autonymie mise en lumière par J. Rey-Debove et les «boucles réflexives » travaillées par J. Authier-Revuz, une modalité principielle du langage et du discours linguistique, et même une autre façon de les investir. C'est sur ce chemin linguistique et sémiotique que nous allons suivre avec passion notre auteure

\section{Dans I'Introduction,}

- l'objet se précise par rétrécissement et distinction entre la "bonne » et la "mauvaise » répétition, entre la répétition exacte et la répétition synonymique, telles qu'elle apparaissent dans les dictionnaires français et allemands : il y aurait une répétition (exacte) à l'identique, caractérisée dans l'expression française "de nouveau», et une répétition (exacte) de la régénérescence, du retour (enrichi) à la première fois, rendue par le français «à nouveau ». La question est bien celle de l'intentionnalité et de la valeur de la répétition, maintenant réduite à la "répétition exacte », lexicale et syntaxique : autrement dit, quand on répète un mot ou un syntagme, l'ajout se fait-il à somme sémantique nulle, en soustraction ou en addition, et quels sont les variables qui nous permettent d'apprécier cette éventuelle valeur? C'est la notion de "répétition figurale", intentionnelle et rechargée sémantiquement, et peut-être même anthropologiquement, qui sera retenue comme objet de l'étude.

- le cadre théorique se dégage peu à peu: une linguistique pré-saussurienne (Bally, p. 31, p. 49) de l'expression et de l'expressivité, benvenistienne de l'énonciation conçue non pas comme le simple appareil de l'énonciation, ni comme les seuls points de vue ou croisements polyphoniques des points de vue, façon A. Rabatel, mais tout autant une gestuelle mentale et affective, proche des plus récentes études cognitivistes, qui met en jeu le corps réel et le corps inconscient, et donc remotive les signes linguistiques tirés de leur conventionnalité. Ainsi une signifiance s'ajouterait à la signification ou la court-circuiterait dans une pragmatique des actes de discours intentionnels. S'esquisse alors une linguistique très 
ambitieuse, de type post-saussurienne (le F. de Saussure des " anagrammes » et de L'Essence double du langage), pouvant peut-être dialoguer avec une poétique du rythme (de type meschonnicien ?) et un cognitivisme des émotions, et pouvant aboutir à une anthropologie du langage déjà amorcée au XIX ${ }^{\mathrm{e}}$ siècle en Allemagne par A. von Humboldt (étonnamment non mentionné par l'auteure). Nous verrons que ce riche cadre théorique s'infléchira peu à peu, notamment vers la Gestalttheorie, ou théorie de la forme, et vers une théorie stylistique (ou néo-stylistique), grandement genettienne quand même, des figures, qui ont leurs avantages, et peut-être leurs limites.

\section{Partie 1 : La répétition figurale} majeures considérées les unes comme obstacles à une théorie anthropologique de la langue, les autres comme échelons progressifs vers une nouvelle théorie plus inclusive, dirait-on aujourd'hui. triptyque: cohésion, cohérence, pertinence, l'un de ces trois concepts dominant successivement les deux autres. C'était oublier le signifiant au profit du seul signifié, c'était valoriser «la toute-puissance du sens conceptuel» au détriment d'une " signifiance perceptive, sensorielle qui ne coïncide pas avec la signifiance ordinaire " (p. 61-62). l'adhésion de l'auteure, car il s'agit bien cette fois d'un arrêt sur le discours, d'un geste de pointage, de la prise en compte partielle de son signifiant et du masque passagèrement posé sur le sens conceptuel «opacifié », et donc sur la non-coïncidence partielle du dit et du dire. E. Prak-Derrington reproche quand même à la théorie de son aînée : la non-prise en compte de la répétition exacte comme : arrêt sur le signifiant, décalage sémantique, poly-énonciation, et iconicité du langage. On pourrait objecter que J. Authier-Revuz, qui n'a pas tout à fait le même objet d'étude, a bien le souci des marques objectives encadrant soit la boucle réflexive, soit le discours citant, comme la ponctuation, le lexique, la syntaxe qui pluralisent le sens et que sa recherche s'ouvre à une théorie plurielle du sujet, faisant même sa place à l'imaginaire. Car toute anthropologie du langage implique une théorie du sujet et du social.

Après quoi l'auteure, laissant de côté la ponctuation notamment, fait une différence entre écrit et oral dans le traitement de la répétition, qui mériterait peut-être d'être nuancée :

La boucle réflexive de la répétition se contente de souligner la modalisation par l'expression répétée, sans donner à l'écrit aucune clé pour l'interpréter. Tout autre est la situation à l'oral, où, si l'imprécision reste programmée, la voix et l'intonation jouent un rôle de premier plan. (p. 64)

Il nous semble que d'autres dimensions des travaux de J. Authier-Revuz, la théorie de P. Fonagy, qui sera bientôt largement invoquée - elle très sensible à la ponctuation et à la poésie, ce qui est bien rare chez les linguistes des trente ou quarante dernières années - et la théorie des figures de la répétition, largement développée par la suite, pourraient nous amener à modérer ce jugement, à proposer quelques "clés " - et finalement à renforcer le corps de la belle thèse défendue. 
17 Cette contestation du signe réduit à son signifié, l'auteure va la retrouver chez A. Rabatel et F. Récanati, avec les phénomènes de sous et de sur-énonciation, dans la linguistique des « anagrammes » de F. de Saussure et de la signifiance de É. Benveniste, lequel recherche dans la poésie - mais dans la poésie séparément, au risque de couper la poésie des autres "genres» de discours - un langage de l'« évocation » et du " sentiment », un peu dans $\mathrm{H}$. Meschonnic avec sa signifiance liée au rythme. On se dit que la psychomécanique guillaumienne aurait pu trouver sa place historique dans cette recherche linguistique pré-cognitive qui essaie de sortir de la fixité du signifié pour trouver une efficacité autre, plus dynamique et plus incarnée du langage, fondée sur une théorie du sujet moins univoque, plus friable.

18 L'auteure évoque, sans trop s'y attarder (p. 79-80), la poésie, qui est pourtant l'un des lieux essentiels de la contestation de la triade signe (réduit au signifié), sens, référence mondaine, même si une juste place est faite à R. Jakobson (pour la prise en compte de l'axe paradigmatique notamment). Avec la poésie c'est en effet, comme pressenti par É. Benveniste, voire F. de Saussure (peut-être parcourus un peu rapidement par notre auteure), une autre signifiance qui s'instaure, moins syntagmatique et linéaire, mais davantage pluralisée, où les instances de l'énonciation deviennent flottantes et potentiellement concurrentes : où se dessine en fait, avec ou sans S. Mallarmé, une autre linguistique, celle que recherche justement E. Prak-Derrington.

19 Le trajet théorique passera aussi par la « lalangue » de J. Lacan, par la phénoménologie de M. Merleau-Ponty, sensible au sujet, au corps immergé et à la parole, par l'« embodiment» de G. Lakoff dans sa théorie de la métaphore, pour rejoindre le cognitivisme et l'«énaction » de F. Varela et de D. Bottineau. On retiendra notamment dans l'endophasie comme dans l'exophasie, la «stimulation directe ou simulée des organes de phonation et de motricité » (p.94). Cet argument cognitiviste qui permet de construire un pont entre écrit et oral, entre lecture silencieuse et lecture oralisée ou proférée, nous semble un argument puissant au service de la future théorie de l'incarnation de notre auteure.

20 Les deux concepts majeurs retenus qui vont tenir maintenant le devant de la scène, seront ceux de forme (ou Gestalt) et de figure :

Je m'inscris explicitement dans la théorie de la Gestalt, ou la psychologie des formes. Pour la Gestalttheorie, les phénomènes ne sont pas perçus de façon atomiste, comme une juxtaposition d'éléments, mais comme des ensembles structurés. (p. 109)

21 Comme dit par l'auteure, sera privilégiée in fine une théorie psychologique de la perception, qui ancre la répétition dans le cognitif et dans le corporel, avec «l'émergence [rastierienne] d'une figure sur un fond» (p. 108), qui fait ainsi saillance. Cette théorie psychologique rencontre une théorie ancienne (et souvent remise en cause depuis soixante ans) des figures rhétoriques où P. Fontanier et G. Genette se rejoignent (p. 109) : la forme globale mise en saillance sera celle de la figure rhétorique sur un fond textuel, mettant en scène et en mouvement l'organisation " réticulaire » (p. 127 sq.) de la répétition.

22 L'une des fonctions de la répétition sera alors de " cohésion rythmique » (p. 137 sq.). Il était logique, pour dépasser la vieille rhétorique, de passer de la forme à la configuration et au rythme, qui impliquent à la fois l'actualisation du discours, le mouvement et sa résonance physique et sémantique. On pourrait se demander toutefois ce qu'apporte à l'expression le mot de "cohésion », alors que le triptyque 
cohésion-cohérence-pertinence semblait mis au second plan dans cette recherche d'une sémantique incarnée. Peut-être le mot « rythme » eût-il suffi : l'ajout du terme de " cohésion » marquerait toutefois une volonté de recourir à une définition du rythme plus stable que celles qui s'étaient proposées à notre esprit. En effet, E. Prak-Derrington ne fait pas grand cas de l'incontournable article d'É. Benveniste sur le «rythmos» (p. 153), glosé tant de fois par les meschonniciens, dont la définition lui semble « purement étymologique» (p. 153), ce qui n'est pas faux et nous avons appris nous aussi à nous méfier de ces justifications si fréquentes par l'étymologie. Celles-ci ne sont jamais des preuves, mais elles peuvent néanmoins être des invitations à penser autrement. La chercheure ne retiendra de la définition benvenistienne que la "forme ", au risque de gommer et le "flux » et la configuration irrégulière du flux, et donc l'apport le plus original de cet article et de son amplification par H. Meschonnic.

On note la même attitude ambiguë face à la théorie de ce dernier. D'un côté :

La notion de rythme est devenue indissociable des travaux de Meschonnic (p. 154)

et de l'autre un rejet définitif, soit pour des raisons bien souvent alléguées par d'autres :

entité sans bords, totalisante, au fondement du sujet, voire de «tous les éléments du discours (ibid)

soit pour des raisons qui pourraient paraître spécieuses ou à contresens :

Chez Meschonnic, le recours constant aux désignations définies (« la langue ", « le sujet », « le discours ») gomme la spécificité de la signifiance (ibid).

Certes, toute théorie conduit à des généralisations (et souvent des essentialisations), mais il est vraiment difficile de reprocher à $\mathrm{H}$. Meschonnic l'estompage de la signifiance alors qu'un de ses concepts-clés est justement celui de "sujet du poème» posé continûment comme « radicalement subjectif ». Cela amène l'auteure à rechercher une définition du rythme plus technique, celle de P. Sauvanet, plus compatible avec le privilège accordé à la théorie plus fixiste de la forme et de la figure.

La définition du rythme par P. Sauvanet est ici d'emblée valorisée : «approche pluridisciplinaire, à dominante esthétique et philosophique », "qui allie puissance synthétique et mobilité », "qui se fonde sur une combinatoire de trois concepts »: "structure, périodicité, mouvement» (p. 155). Des trois concepts il suffirait que deux soient réunis pour qu'il y ait rythme : le deux sur trois ne nous rassure pas vraiment. De ceux-ci, l'auteure, nolens volens, privilégie celui de « périodicité » au sens de H. Morier : « retour à intervalles sensiblement égaux, d'un repère constant » (Morier, 1998) (ibid.), marquant ainsi l'ancrage de sa théorie dans la rhétorique plus statique des figures et son choix de rythme comme régularité, ce qui rendrait probablement les deux critères d'accompagnement, structure et mouvement, ancillaires. Cela se traduira en effet, dans le cours de la démonstration, par la dissolution, au moins partielle, des notions de structure et de mouvement, dans les tropes. Nous admettons bien que la notion de répétition d'un élément plusieurs fois (de deux à sept ou huit occurrences) imposait une forme de régularité, de parallélisme ou de symétrie - ce qui n'est au fond qu'un type de rythme (ou peut-être une de ses composantes) parmi d'autres, relevant peutêtre plus que d'autres d'un imaginaire collectif, et d'un acte de langage que notre auteure pourra qualifier ensuite.

29 On aboutit logiquement à une classification conventionnelle des figures dites rythmiques de la répétition : anaphore, épanode, épiphore, symploque, antépiphore, 
anadiplose, chiasme (p. 157-159) et à leur illustration dans des exemples tirés du champ politique. Même si cet aboutissement à la figuralité stylistique la plus ancienne a de quoi surprendre, l'auteure fait deux avancées majeures : ces figures, souvent étudiées séparément sont ici regroupées dans cet ensemble englobant de la répétition; et surtout elle met en valeur l'iconicité du langage, où « le son précède le sens » : la figure sonore, orale ou écrite, n'est pas un simple arrangement argumentatif et persuasif du sens, mais un outil de production du sens, autrement dit une "machine». De même, dirions-nous à notre tour, le début d'une phrase n'est pas seulement une partie de phrase déjà constituée, mais l'amorce d'un discours qui se cherche encore, rythmiquement et sémantiquement ; le fameux exemple de M. L. King, I have a dream discours en partie improvisé, précise E. Prak-Derrington - en sera un exemple fort bien étudié dans la seconde partie de l'ouvrage. Où la figure participe à une phénoménologie de la parole en mouvement, orale et peut-être écrite, qui peut en outre se raccorder à certaines études cognitives récentes des tropes (certes évoquées, mais qui auraient mérité un petit développement).

Dans ce mouvement de la parole, le visuel et le sonore, amarrés au signifiant, jouent un rôle qui n'est plus seulement stylistique ni à proprement parler poétique, mais cognitif, poïétique et communicatif, pour ne pas dire émotionnel. C'est sur ce terreau refertilisé que se construit ce qui sera appelé bientôt «la modalité de répétition » du langage, refaisant boucle avec Bally (p. 183), et probablement le dépassant.

\section{II - Partie 2 : La répétition performative (p. 185 - 334)}

31 La seconde partie, de même longueur que la première, récoltera les fruits du long cheminement théorique vers des figures animées, "mimologiques », autoproductrices de discours et d'une nouvelle relation à l'allocutaire. La nouvelle détermination de la répétition comme "performative " nous ouvre à une physique et à une pragmatique des figures et des discours, où la notion austinienne et searlienne de performativité est assumée bien au-delà de certaines formules répétées à l'envie, je le jure, par tous les linguistes, pour aborder à une performativité généralisée du langage : un « répéter c'est faire» (p. 192 et seq.). Et ce sera même plus que faire, nous le verrons, car «la dimension corporelle et gestuelle du langage » engage une autre relation à autrui, peutêtre même au cosmos. " "Et la parole a été faite chair" (Jean, I, 14)» (p. 197). Ici commence la «magie de la répétition, [la] magie de l'incarnation » (ibid.).

Notre auteure s'attache d'abord à la litanie religieuse ou politique et à la chanson populaire où s'actualisent de façon privilégiée les figures de la répétition. La litanie met en déroute les règles conversationnelles de $\mathrm{P}$. Grice (informativité et exhaustivité) et la notion de pertinence de D. Sperber et D. Wilson au profit d'un "méta-acte" performatif $d u$ «montrer-dire» (p. 211), qui s'appuie sur le retour autonymique, tout en retrouvant J. Authier-Revuz. L'énonciateur (orateur, prêcheur, slameur, chanteur de gospel ou autre) passe alors d'une position singulière à une position générale et collective de « surlocuteur ", qui englobe l'allocutaire et le généralise à son tour, avant de le promouvoir en énonciateur-réénonciateur. La «scène litanique » comme "scène rituelle» se marque alors par son "iconicité référentielle [...], énonciative[...], pragmatique[...] et interactionnelle » (p. 240).

Au chapitre 6, E. Prak-Derrington examine (à partir de la page 243) 
la relation entre la performance, pensée comme iconicité entre celui ou celle qui parle et ceux ou celles qui l'écoutent, et la performativité, pensée comme iconicité entre corporéité et acte effectué. oral et écrit] s'est aujourd'hui largement imposé ", citant C. Blanche-Benveniste et le GARS. C. Blanche-Benveniste voulait avant tout construire une linguistique de l'oral, distincte, et a été confrontée, avec le groupe de recherche d'Aix-en-Provence sur l'oral (le GARS) au problème de la transcription (Deulofeu). Il nous semble qu'est encore très vivace, du côté de la Suisse et ailleurs, une linguistique des unités de discours binaire, où l'unité discursive fondamentale de l'écrit serait la phrase (Benveniste), tandis que celle de l'oral serait la période (Berrendonner). Il est vrai que A. Berrendonner passe souvent de l'oral à l'écrit et a tendance - parfois exagérément - à analyser l'écrit avec ses concepts de l'oral. Alors que peut-être, en effet, phrase et période sont-elles des unités opérant dans les deux régimes, mais avec des dominantes différentes. On notera que l'auteure n'évoque presque jamais ces questions d'unités discursives, pourtant essentielles, la répétition lexicale et donc aussi phonique entrainant toute une accentuation et une mise au jour d'«unités fluctuantes" (M. Favriaud après G. Dessons), non linéaires, qui jouent avec les unités linéaires de phrase, période, paragraphe et texte, et participent étroitement au rythme (irrégulier autant que régulier) et à la dynamique de la signifiance, qui, ainsi, ne viendrait pas uniquement de l'« incarnation » physique et vocale.

Le long hommage rendu à $\mathrm{P}$. Fonagy comme précurseur nous semble d'autant mieuxvenu que la recherche monumentale de ce dernier nous semble souvent ignorée de nos contemporains. On reprendra volontiers cette partie de la citation du linguistephonéticien-psychanalyste hongrois (p. 253):

Il est impossible de faire fonctionner ces organes sans qu'ils puissent s'exprimer à

leur tour, en ajoutant au message linguistique des informations de nature

différente. (Fonagy 1983, p. 23)

Les marques de ponctuation à l'écrit si l'on pratique une théorie de la " ponctuation étendue " (M. Favriaud) seraient capables de rendre compte en partie, mais en partie seulement, de la triple implication du corps, de l'imaginaire subjectif et de la réflexivité dans le discours écrit (M. Favriaud après P. Fonagy justement). D'ailleurs d'autres psychanalystes que P. Fonagy se sont intéressés à la ponctuation comme lieu intermédiaire entre conscient et inconscient, susceptible de contribuer à l'« empathie " relevée par notre auteure, comme "sixième sens" (à la suite de M.-L. Brunel et J. Cosnier) (p. 260). 

de mieux comprendre les phénomènes d'échoïsation, de co-énonciation et d'aphorisation des énoncés répétés, et de ce qu'on pourrait appeler, dans le sillage de l'auteure, une « co-incarnation » ou une « incarnation croisée » des discours.

-Derrington termine son développement par deux chapitres consacrés à la « réduplication » et à l'« incantation » comme deux formes privilégiées de la modalité de l'incarnation» (p. 273).

La réduplication est la forme la plus banale et la plus étudiée de répétition. (ibid)

C'est le fait de répéter, et de manière exacte et à proximité immédiate, une unité

linguistique : phonème, syllabe, mot, syntagme, voire énoncé (p. 274)

La réduplication serait donc un type de répétition exacte concernant des unités d'empan très variable, mais en succession immédiate. Ici notre auteure paie son écot reconnaissant à E. Richard et M.-A. Watine (p. 282 et seq.) On la trouve d'abord dans le babil enfantin, bobo, dodo, papa, mama, et dans la réponse échoïque des parents proches, utilisant le parler-bébé, au «caractère préverbal et multimodal» (p. 282) ; ensuite dans le discours, surtout oral ou mimétique de l'oral, comme dans cet exemple : «Félix est parfait, parfait, parfait!» (p. 283). E. Richard l'interprète comme un retour sur l'énonciation marqué par un "haut degré de charge énonciative » (ibid) à valeur connotative, tandis que M.-A. Watine insiste sur sa « dimension méta-énonciative » soit de confirmation soit de distanciation du dit par le dire. Pour l'auteure lyonnaise, la réduplication est prise dans un faisceau multimodal qui lui donne sens en contexte; c'est un geste vocal, verbal et corporel où la ponctuation jouerait un rôle à l'écrit, et l'intonation à l'oral et même à l'écrit (p. 287), ce qui nous ramène à P. Fonagy. Ne pourrait-on pas aller jusqu'à proposer à l'auteure la notion de ponctuation mimogestuelle de l'oral dont le « blank $^{1}$ face » S. d'Attardo (le visage neutre) (p. 296) serait un élément? Ainsi

la réduplication re-motive, re-donne corps au signe utilisé, en faisant appel à l'oreille, mais aussi aux yeux (aspect kinésique : sourire, froncement de sourcils, mouvement des yeux, de la main, etc.) (p. 290)

41 L'analyse détaillée de ce geste verbo-corporel sera conduite dans le savoureux examen $\mathrm{du}$ sketch de la standardiste avec Y. Montand, l'amoureux, et S. Signoret, la standardiste impassible, répétant tour à tour, et chacun à sa façon, le « je t'aime, je t'aime, je t'aime ", puis dans une diatribe enflammée dont A. Finkielkraut a le secret, ici avec A. Haenel sur le féminisme, avec un tonitruant « violez, violez, violez » où, selon l'auteure, la parole ironique et le geste mimo-corporel du polémiste auraient été en discordance, provoquant un qui pro quo propice au scandale. Le découplage de la parole et du geste montre, par contraste, le nouage habituel indissoluble des deux.

Enfin E. Prak-Derrington pousse l'acmé de sa démonstration jusqu'à l'« incantation », religieuse, politique ou littéraire, où la simple nomination réitérée, chantée, déclamée, celle du nom propre ou de la formule - loin de tout développement discursif ou argumentatif et de toute falsification et vériconditionnalité - fait accéder le discours remotivé par la voix et le corps et pour ainsi dire réasserté à l'envi, à une performativité redoublée. Les interlocuteurs sont amenés (contraints ?) à une intensification de l'attention, à une communion (p.344), voire à une transe de communication émotionnelle.

43 Concluons en notant d'abord l'immense bibliographie de l'auteure qui montre non seulement un riche savoir de sciences du langage et de sciences humaines, mais aussi 
un dévouement, presque une dévotion, au sujet qu'elle parcourt brillamment et qu'elle fait avancer sur des points notables. Le lecteur la suivra avec la même passion, même si, à certaines pattes d'oie, il aurait pris le chemin de gauche plutôt que celui de droite emprunté par l'auteure, et vice versa. En cela, l'exposé de cette théorie est ouvert.

En tout cas, la cible de l'auteure semble atteinte : faire sortir la répétition et sa souscatégorie de réduplication de leur placard mal famé, montrer non seulement leur fonctionnement et leur efficace (performative, peut-être pas « magique » quand même) mais aussi l'autre face du langage qu'elle contribue à révéler : un langage qui se soucie moins de la signification conceptuelle déjà-là et de communication univoque, au demeurant indéfinissable, que de la signifiance prise dans un nouage inextricable avec le corps, la voix, le geste - et probablement l'imaginaire, singulier ou collectif, probablement plus collectif que singulier d'ailleurs - où les sujets non seulement échangent des rôles d'énonciation mais se transforment: se complexifient ou au contraire - prenons-y garde - pourraient se soumettre. Gare au «corps collectif » et à cet « embrayeur sur l'extraordinaire» (p. 344), gare à l'incarnation et à l'incantation qui seraient sans réflexivité ni éthique, car dans la répétition le " posé » devient vite le "présupposé », et change de régime de vérité (p. 349)!

E. Prak-Derrington faisant retour vers une des meilleures alliées de ce pèlerinage heuristique, J. Authier-Revuz, pose cette question inaugurale de nouvelles recherches (p. 348):

Le DD [discours direct] nous confronte aux modalités corporelles (et aux pouvoirs) de la profération. [...] Le discours direct pourrait-il se définir, au-delà de sa matérialité autonymique, comme la modalité de l'incarnation de la parole rapportée?

Cette recherche est ouvrante non seulement par ce qu'elle démontre - d'un tiroir du langage donné à trier et explorer méthodiquement - mais aussi par les questions qu'elle pose à tout le langage. N'y a-t-il pas, bien au-delà de la répétition des textes épidictiques, politiques et religieux, bien au-delà même de la poésie et de toute la littérature, non seulement un « méta-acte de répétition » mais aussi toute une physique $\mathrm{du}$ langage et de l'interaction langagière, que les théories de la phonation, de l'énonciation puis de la cognition liée aux émotions nous faisaient entrevoir? Les mots d'incarnation, de geste vocal, de motivation iconique des signes, de multimodalité langagière et sémiotique - auxquelles on pourrait ajouter ceux de syntaxe et de sémantique plurielles - nous font accéder à une nouvelle anthropologie du langage, de la vérité et de l'éthique, du rapport entre langage et monde.

Et en didactique, chère à Pratiques, ajouterons-nous, à quelques bouleversements potentiels : la répétition de l'énoncé et des gestes d'apprentissage (on notera l'intrusion à l'école primaire depuis une quinzaine d'années des "rituels » d'apprentissage) n'est-elle pas globalement minorée ? Et la frontière entre écrit et oral souvent adornée de barbelés? Quelle place faire à la répétition de mémorisation? Quelles sont les activités qui permettraient cette incarnation du langage, reliée éthiquement - à la nécessaire réflexivité? Une didactique du Français langue maternelle et du Français langue étrangère appuyée sur le théâtre, l'écriture et la représentation, pourraient peut-être constituer une petite partie de la réponse.

Donc, les concepts mis au jour ou renforcés par E. Prak-Derrington sont un nouvel outil qui prend sa place, historique, dans la reconstruction en cours des sciences du langage et de leur dialogue possible avec la poétique des formes et langages littéraires, 
l'anthropologie et les sciences humaines, et les sciences cognitives devenues plus matures et plus ouvertes aux questions linguistiques, philosophiques, artistiques. La linguistique montre - depuis un certain temps déjà, et le mouvement néo-saussurien en est le navire-amiral en francophonie depuis une bonne cinquantaine d'années - qu'elle a atteint un niveau de maturité tel qu'elle peut se remettre, partiellement, en question. La description critique du langage pris dans toute sa complexité et sa saveur a bien sa place dans la construction culturelle et sociale en ce moment de vacillement des démocraties. Ce gros livre, qui s'enjoue de la répétition, fait du bien, ouvre sur l'avenir, et donne aussi à réfléchir par les belles questions qu'il suscite.

\section{NOTES}

1. N'oublions pas que «blank» est utilisé aussi en anglais pour signifier le blanc de ponctuation, ou « ponctuation blanche ». 